\section{Theoretical \& Applied Science}

p-ISSN: 2308-4944 (print) e-ISSN: 2409-0085 (online)

Year: $2018 \quad$ Issue: $01 \quad$ Volume: 57

Published: $30.01 .2018 \quad$ http://T-Science.org

SECTION 28. Pharmaceutical Sciences. reseacher

Pyatigorsk medical-pharmaceutical Institute-branch state budget educational institution of higher professional education "Volgograd State Medical University" Ministry of healthcare of the Russian Federation

\section{V.A. Morozov}

reseacher

Pyatigorsk medical-pharmaceutical Institute-branch state budget educational institution of higher professional education "Volgograd State Medical University" Ministry of healthcare of the Russian Federation

M.K. Tsidaeva postgraduate

Pyatigorsk medical-pharmaceutical Institute-branch state budget educational institution of higher professional education "Volgograd State Medical University" Ministry of healthcare of the Russian Federation, Pyatigorsk

\title{
DEVELOPMENT OF A COMPLEX SOFTWARE AS A WAY OF OPTIMIZATION OF DRUG PROVISION OF THE POPULATION
}

\author{
Abstract: Medicinal maintenance of the population is one of the challenges facing practical pharmacy. Under \\ this is extremely important to have clear and reliable definition of medicines requirements. \\ Key words: Pharmacoeconomics, pharmacoepidemiology, drug supply of the population, drug supply of rural \\ population, pharmaceutical care. \\ Language: Russian \\ Citation: Airo IN, Morozov VA, Tsidaeva MK (2018) DEVELOPMENT OF A COMPLEX SOFTWARE AS \\ A WAY OF OPTIMIZATION OF DRUG PROVISION OF THE POPULATION. ISJ Theoretical \& Applied \\ Science, 01 (57): 133-136. \\ Soi: http://s-o-i.org/1.1/TAS-01-57-24 Doi: crossef https://dx.doi.org/10.15863/TAS.2018.01.57.24

\section{РАЗРАБОТКА КОМПЛЕКСА ПРОГРАММНОГО СОФТА КАК ОДИН ИЗ СПОСОБОВ ОПТИМИЗАЦИИ ЛЕКАРСТВЕННОГО ОБЕСПЕЧЕНИЯ НАСЕЛЕНИЯ}

Аннотация: Лекарственное обеспечение населения является одной из задач стоящих перед практической фармачией. В рамках этого представляется исключительно важным четкое и достоверное определение потребностей в лекарственных средствах

Ключевые слова: Фармакоэкономика, фармакоэпидемиология, лекарственное обеспечение населения, лекарственное обеспечение населения сельской местности, фармацевтическая помощь.

\section{Introduction}

В настоящее время одной из актуальных проблем, стоящих перед российским здравоохранением, является качественное и своевременное лекарственное обеспечение населения.

Изменения показателей заболеваемости населения и изменения в номенклатуре лекарственных препаратов, а также появление новых терапевтических схем лекарственной терапии различных заболеваний приводят к изменению структуре потребления ЛС.

Если учесть рост числа больных хроническими заболеваниями и быстрое старение населения, легко сделать вывод, что никогда еще потребность в повышении качества медицинских услуг и снижении соответствующих расходов не была такой острой.

Однако, чаще приходится сталкиваться с недостатком лекарственных средств в больницах, стационарах, нехваткой медикаментов для больных социально-значимыми заболеваниями.

По мнению ведущих исследователей и руководящих кадров Министерства Здравоохранения РФ зачастую причиной случаев задержек отпуска лекарственных средств являются именно погрешности, допущенные при определении потребностей, как один из факторов 


\begin{tabular}{|c|c|c|c|c|c|c|}
\hline Impact Factor: & $\begin{array}{l}\text { ISRA (India) } \\
\text { ISI (Dubai, UAE } \\
\text { GIF (Australia) } \\
\text { JIF }\end{array}$ & $\begin{array}{r}=1.344 \\
=0.829 \\
=0.564 \\
=1.500\end{array}$ & $\begin{array}{l}\text { SIS (USA) } \\
\text { PИНЦ (Russia) } \\
\text { ESJI (KZ) } \\
\text { SJIF (Morocco }\end{array}$ & $\begin{array}{l}=0.912 \\
=0.207 \\
=4.102 \\
=\mathbf{2 . 0 3 1}\end{array}$ & $\begin{array}{l}\text { ICV (Poland) } \\
\text { PIF (India) } \\
\text { IBI (India) }\end{array}$ & $\begin{array}{l}=6.630 \\
=1.940 \\
=4.260\end{array}$ \\
\hline
\end{tabular}

увеличения смертности и высокой инвалидизации, неудовлетворенности населения качеством оказания медицинской помощи.

Здравоохранение как минимум на десятилетие отстает от других отраслей по уровню освоения информационных технологий, позволяющих повысить качество и эффективность работы.

В рамках этого представляется исключительно важным четкое и достоверное определение потребностей в лекарственных средствах, что служит основой для своевременного и бесперебойного лекарственного обеспечения населения в учреждениях здравоохранения.

Для решения данной проблемы нами разработан программный алгоритм и на его основе планируется создание программного обеспечение для прогнозирования потребления лекарств.

Кроме того, немаловажным является возможность упрощения обращения с программой, т.е. создание такого интерфейса, который бы позволил пользователю, зачастую не обладающему математическими навыками пользоваться программой.

С помощью программы будет возможным прогнозирование потребностей в лекарственных препаратах для учреждений здравоохранения, а также определение финансовых затрат, что приведет к более правильному расходованию денежных средств государства, а также бесперебойному обеспечению населения медикаментами.
В качестве метода расчета был выбран подход, позволяющий сочетать в себе при расчетах нормативный метод и методы математического моделирования процесса заболеваемости.

Расчет основывается на изучении заболеваемости по нозологическим формам, структуре потребления препаратов, а также количественном расходе при назначении больному, в том числе одновременном, что является особенно важным моментом, так как больные зачастую получают терапию несколькими препаратами.

Так же с помощью математического моделирования будет учитываться динамика изменения показателей заболеваемости.

\section{Materials and Methods}

Интерфейс компьютерной программы предусматривает два рабочих окна - «Данные» и «Прогнозирование», что обеспечивает параллельное использование программы для расчета потребностей в лекарственных средствах и для проведения фармакоэпидемиологических исследований.

В табл. 1 и 2 представлена техническая значимость проекта, сравнительный анализ программы с существующим способом прогнозирования потребления ЛС и формирования заявки, а также представлены преимущества и недостатки программы.

Табл. 1

Сравнительный анализ программы с существующим способом прогнозирования потребности.

\begin{tabular}{|c|c|c|}
\hline Характеристики & $\begin{array}{c}\text { Существующий способ } \\
\text { прогнозирования и } \\
\text { формирования заявки }\end{array}$ & $\begin{array}{c}\text { Программа для расчета } \\
\text { потребности в ЛС }\end{array}$ \\
\hline Оптимизация затрат денежных средств & - & + \\
\hline $\begin{array}{l}\text { Точное прогнозирование потребности } \\
\text { в ЛС и составление заявки }\end{array}$ & - & + \\
\hline $\begin{array}{l}\text { Принятие } \\
\text { упоснованных } \\
\text { управленческих решений в области } \\
\text { лекарственного обеспечения }\end{array}$ & - & + \\
\hline $\begin{array}{l}\text { Формирование базы данных о } \\
\text { потреблении лекарственных средств } \\
\text { больными различными патологиями за } \\
\text { ряд лет }\end{array}$ & - & + \\
\hline Автоматизация процесса & - & + \\
\hline
\end{tabular}


Таким образом представленный софт улучшает современную технологию расчета потребности в лекарственных препаратах будущих периодов как в рамках отдельно взятого учреждения здравоохранения, а также региона и Российской Федерации

Табл. 2

\section{Техническая значимость проекта}

\begin{tabular}{|c|c|c|}
\hline Свойства программы & $\begin{array}{c}\text { Преимущества использования } \\
\text { программы }\end{array}$ & Выгода / Результат \\
\hline Точность расчетов программы & $\begin{array}{l}\text { Исключает ошибку и } \\
\text { человеческий фактор при } \\
\text { формировании потребности }\end{array}$ & $\begin{array}{l}\text { Правильное расходование } \\
\text { денежных средств }\end{array}$ \\
\hline $\begin{array}{l}\text { Автоматическое формирование } \\
\text { заявки }\end{array}$ & Ускоряет процесс работы & $\begin{array}{l}\text { Своевременное обеспечение } \\
\text { больных необходимыми } \\
\text { медикаментами }\end{array}$ \\
\hline Поливариантный расчет прогноза & $\begin{array}{l}\text { Использование программы для } \\
\text { всех групп лекарственных } \\
\text { препаратов с учетом темпа } \\
\text { прироста показателей } \\
\text { заболеваемости }\end{array}$ & $\begin{array}{l}\text { Повышает техническую } \\
\text { значимость программы }\end{array}$ \\
\hline $\begin{array}{l}\text { Удобный и универсальный } \\
\text { интерфейс программы }\end{array}$ & $\begin{array}{l}\text { Легкость использования } \\
\text { программы }\end{array}$ & $\begin{array}{l}\text { Позволяет использование } \\
\text { программы людям с } \\
\text { минимальными навыками в } \\
\text { математическом прогнозировании }\end{array}$ \\
\hline $\begin{array}{l}\text { Возможность архивирования, } \\
\text { сохранения и обработки } \\
\text { информации }\end{array}$ & $\begin{array}{l}\text { Позволяет накапливать } \\
\text { информацию прошлых периодов } \\
\text { для более точного } \\
\text { прогнозирования }\end{array}$ & $\begin{array}{l}\text { Снижение ошибки } \\
\text { прогнозирования потребности в } \\
\text { лекарственных препаратах }\end{array}$ \\
\hline
\end{tabular}

\section{Conclusion}

Недостатком программы является отсутствие изначальной интеграции в систему здравоохранения России.

Данный проект потенциально интересен Министерству Здравоохранения Российской Федерации и фармацевтическим организациям, и может быть использован на уровне региона, лечебно-профилактического учреждения или его отделения.

Данное программное обеспечение должно позволить использовать его в любых необходимых объемах.

\section{References:}

1. (2018) Diabet. Available: http://www.diabetunet.ru/diabetes-risk-factors (Accessed: 10.01.2018).

2. Arkhangel'skiy A.Ya. Programmirovanie v $\mathrm{C}++$ Builder.

(2010) 


\begin{tabular}{|c|c|c|c|c|c|c|}
\hline Impact Factor: & $\begin{array}{l}\text { ISRA (India) } \\
\text { ISI (Dubai, UAE } \\
\text { GIF (Australia) } \\
\text { JIF }\end{array}$ & $\begin{array}{r}=1.344 \\
=0.829 \\
=0.564 \\
=1.500\end{array}$ & $\begin{array}{l}\text { SIS (USA) } \\
\text { PИНЦ (Russia) } \\
\text { ESJI (KZ) } \\
\text { SJIF (Morocco) }\end{array}$ & $\begin{array}{l}=0.912 \\
=0.207 \\
=4.102 \\
=2.031\end{array}$ & $\begin{array}{l}\text { ICV (Poland) } \\
\text { PIF (India) } \\
\text { IBI (India) }\end{array}$ & $\begin{array}{l}=6.630 \\
=1.940 \\
=4.260\end{array}$ \\
\hline
\end{tabular}

5. Gribova Ya.V. (2011) Osobennosti prognozirovaniya potrebnostey v lekarstvennykh sredstvakh / Ya.V. Gribova // Vestnik Kazanskogo tekhnologicheskogo universiteta. - 2011. № 1 - p. 171-174.

6. Dremova N.B. (1999) Komp'yuternye tekhnologii marketingovykh issledovaniy $\mathrm{v}$ meditsinskikh i farmatsevticheskikh organizatsiyakh: ucheb.- metod. posobie / N.B. Dremova, S.V. Solomka. - Kursk: KGMU, 1999. - $150 \mathrm{p}$.

7. Dremova N.B. (2008) Problemy farmatsevticheskogo menedzhmenta $\mathrm{V}$ lechebno-profilakticheskom uchrezhdenii / N.B. Dremova, I.V.Tolkacheva // Farmatsiya. 2008. № 5. - p. 37-42.
8. Morozov V.A. (2005) Metodicheskie rekomendatsii po sovershenstvovaniyu lekarstvennogo obespecheniya bol'nykh sakharnym diabetom Respubliki Severnaya Osetiya-Alaniya / V.A. Morozov, V.L. Bazarnyy. - Kursk: Izd-vo Kurskaya gorodskaya tipografiya, 2005. - 76 p.

9. Dubrova T.A. (2004) «Statisticheskie metody prognozirovaniya v ekonomike», 2004

10. Umarov, S.Z. (2002) Analiz otechestvennogo opyta normirovaniya potrebleniya lekarstvennykh sredstv / S.Z. Umarov, Yu.V. Miroshnichenko, I.A. Narkevich. // Ekonomich. vestn. farmatsii. - 2002. - № 6. - p. 75-79. 